\title{
Targeting Dys-Regulated Reactive Oxygen Species Homeostasis in Cancer as a Selective Chemotherapeutic Approach
}

\section{Danuta S. Kalinowski ${ }^{1 *}$ and Des R. Richardson ${ }^{1}$ \\ Iron Metabolism and Chelation Program, Department of Pathology, Bosch Institute, University of Sydney, Sydney, Australia}

The development of resistance by cancer cells to established chemotherapeutics is a key issue in the clinical treatment of this devastating disease and has necessitated the generation of therapeutic agents that utilize novel targeting strategies [1]. Identifying crucial biochemical alterations between cancer cells and their normal counterparts is critical in the design of potent chemotherapeutics that maintain selectivity and overcome drug resistance [2]. Importantly, the increase in oxidative stress and reactive oxygen species (ROS) generation observed in cancer cells, in comparison to their normal counterparts, has been highlighted as a possible selective targeting strategy [2]. Thus, the use of agents that are able to modulate levels of intracellular ROS in oxidatively stressed cancer cells forms a potential chemotherapeutic approach.

In normal cells, the maintenance of ROS homeostasis is essential for survival and growth [2]. In fact, ROS are essential in biological signaling transduction pathways and the regulation of enzyme activity, such as ribonucleotide reductase (RR) [3]. Importantly, RR is an ironcontaining enzyme involved in the rate-limiting step of DNA synthesis that has previously acted as a chemotherapeutic target for hydroxyurea [1]. While moderate levels of ROS promote cellular proliferation and differentiation, excessive ROS levels are toxic and can result in oxidative damage to vital biomolecules, including DNA, proteins and lipids [1]. Thus, ROS homeostasis is achieved by balancing ROS production and elimination that occurs through endogenous scavenging systems such as catalase, thioredoxin and superoxide dismutase [2,3].

In vitro and in vivo studies and clinical specimens have demonstrated that neoplastic cells function with increased levels of oxidative stress compared to normal cells [4-7]. This is evident in the increased levels of oxidation products, such as lipid peroxides and oxidized DNA bases in cancer cells $[7,8]$ and is thought to result from factors including mitochondrial dysregulation and activation of oncogenes [9-11]. Furthermore, levels of ROS-scavenging molecules and enzyme systems have been previously assessed in primary and malignant tumors (e.g. colorectal, lung and ovarian cancer) and compared to normal noncancerous tissue [12-16]. Interestingly, the majority of cancer types show an imbalance in anti-oxidant enzyme levels in comparison to the cell of origin [14]. For example, decreased levels of radical-scavenging systems (e.g. reduced glutathione, glutathione peroxidase 1 and 3, and glutathione reductase) have been observed in a number of cancer types (e.g. colorectal cancer, glioblastoma multiforme and transitional meningioma), in comparison to normal tissue $[13,14,16]$. In contrast, ovarian cancers display increased levels of superoxide dismutase and this increase is believed to be a cellular response to increased ROS levels [12]. These factors indicate a dys-regulation in ROS homeostasis in tumors [12-15] and suggest that cancer cells are more susceptible than normal cells to insult by agents that further disrupt ROS homeostasis. Thus, exogenous agents that increase intracellular ROS levels, such as iron chelators that form redox active complexes $[17,18]$, are an exciting chemotherapeutic avenue.

Interest in thiosemicarbazone iron chelators as anti-cancer agents has increased in recent years, with the ligand, Triapine, entering $>20$ international clinical trials [19]. Additionally, a number of thiosemicarbazone analogues, including di-2-pyridylketone 4,4-dimethyl-3-thiosemicarbazone (Dp44mT) and 2-benzoylpyridine 4,4-dimethyl-3-thiosemicarbazone (Bp44mT), overcome resistance to established chemotherapeutics and have demonstrated potent and selective anti-cancer activity against a number of human tumor xenografts in vivo [20,21].

Importantly, the anti-proliferative activity of thiosemicarbazones is not only due to the chelation of the essential nutrient, iron, but also results from the formation of redox-active iron complexes $[17,18]$. Once formed, these iron complexes are able to participate in Fenton-like chemistry to generate ROS and can interact with cellular oxidants and reductants, establishing a catalytic redox cycling mechanism $[1,17,18]$. Significantly, Dp44mT and Bp44mT were shown to alter important thiol-related anti-oxidant systems in vitro, including glutathione, thioredoxin and glutaredoxin [3]. In fact, these iron chelators significantly increased the levels of oxidized trimeric thioredoxin and was most likely due to their ability to inhibit thioredoxin reductase activity [3]. Moreover, these ligands also resulted in the inhibition of glutathione reductase activity, which is responsible for the generation of reduced glutathione, an important cellular anti-oxidant [3]. Such thiol-containing systems provide reducing equivalents for $R R$, the enzyme involved in the rate-limiting step of DNA synthesis [3]. Thus, targeting these systems also reduce the activity of RR [3] and these studies provide further insight into the mechanisms involved in the anti-cancer activity of thiosemicarbazones [3].

Collectively, agents that can modulate levels of intracellular ROS, either through the increase in ROS generation and/or the inhibition of ROS-scavenging capacity, form a potential therapeutic avenue to selectively target cancer [2]. In this light, novel thiosemicarbazone iron chelators, such as Dp44mT and Bp44mT, have emerged as potent and selective anti-cancer agents that act via multiple mechanisms, including: (1) the chelation of iron from biological systems $[1,17,18]$; (2) the formation of redox active iron complexes $[1,17,18]$; and (3) the alteration of thiol-containing anti-oxidant systems to inhibit RR activity [3]. Significantly, the targeting of multiple cellular systems is an important strategy in design of selective agents that overcome resistance to established chemotherapeutics. The ability of these chelators to target cellular anti-oxidant systems, which are already compromised in many cancer types, may explain the potent and selective anti-cancer activity of these ligands observed in vivo [20,21].

\section{References}

1. Kalinowski DS, Richardson DR (2005) The evolution of iron chelators for the treatment of iron overload disease and cancer. Pharmacol Rev 57: 547-583.

${ }^{*}$ Corresponding author: Dr. Danuta S. Kalinowski, Department of Pathology, Bosch Institute, University of Sydney, Sydney, New South Wales, 2006 Australia, Tel: +61-2 90366547; Fax: +61-2-93513429; E-mail: danuta.kalinowski@sydney.edu.au

Received April 12, 2012; Accepted April 12, 2012; Published April 16, 2012

Citation: Kalinowski DS, Richardson DR (2012) Targeting Dys-Regulated Reactive Oxygen Species Homeostasis in Cancer as a Selective Chemotherapeutic Approach. Vitam Trace Elem 1:e113.

Copyright: (C) 2012 Kalinowski DS, et al. This is an open-access article distributed under the terms of the Creative Commons Attribution License, which permits unrestricted use, distribution, and reproduction in any medium, provided the original author and source are credited. 
Citation: Kalinowski DS, Richardson DR (2012) Targeting Dys-Regulated Reactive Oxygen Species Homeostasis in Cancer as a Selective Chemotherapeutic Approach. Vitam Trace Elem 1:e113.

2. Trachootham D, Alexandre J, Huang P (2009) Targeting cancer cells by ROSmediated mechanisms: a radical therapeutic approach? Nat Rev Drug Discov 8: $579-591$.

3. Yu Y, Suryo Rahmanto Y, Hawkins CL, Richardson DR (2011) The potent and novel thiosemicarbazone chelators di-2-pyridylketone-4,4-dimethyl-3thiosemicarbazone and 2-benzoylpyridine-4,4-dimethyl-3-thiosemicarbazone affect crucial thiol systems required for ribonucleotide reductase activity. Mol Pharmacol 79: 921-931.

4. Szatrowski TP, Nathan CF (1991) Production of large amounts of hydrogen peroxide by human tumor cells. Cancer Res 51: 794-798.

5. Kamiguti AS, Serrander L, Lin K, Harris RJ, Cawley JC, et al. (2005) Expression and activity of NOX5 in the circulating malignant B cells of hairy cell leukemia. $\mathrm{J}$ Immunol 175: 8424-8430.

6. Zhou Y, Hileman EO, Plunkett W, Keating MJ, Huang P (2003) Free radica stress in chronic lymphocytic leukemia cells and its role in cellular sensitivity to ROS-generating anticancer agents. Blood 101: 4098-4104.

7. Kumar B, Koul S, Khandrika L, Meacham RB, Koul HK (2008) Oxidative stress is inherent in prostate cancer cells and is required for aggressive phenotype. Cancer Res 68: 1777-1785.

8. Patel BP, Rawal UM, Dave TK, Rawal RM, Shukla SN, et al. (2007) Lipid peroxidation, total antioxidant status, and total thiol levels predict overall survival in patients with oral squamous cell carcinoma. Integr Cancer Ther 6 : 365-372.

9. Rodrigues MS, Reddy MM, Sattler M (2008) Cell cycle regulation by oncogenic tyrosine kinases in myeloid neoplasias: from molecular redox mechanisms to health implications. Antioxid Redox Signal 10: 1813-1848.

10. Brandon M, Baldi P, Wallace DC (2006) Mitochondrial mutations in cancer Oncogene 25: 4647-4662.

11. Irani K, Xia Y, Zweier JL, Sollott SJ, Der CJ, et al. (1997) Mitogenic signaling mediated by oxidants in Ras-transformed fibroblasts. Science 275: 1649-1652.

12. Hu Y, Rosen DG, Zhou Y, Feng L, Yang G, et al. (2005) Mitochondrial manganese-superoxide dismutase expression in ovarian cancer: role in cell proliferation and response to oxidative stress. J Biol Chem 280: 39485-39492.

13. Murawaki Y, Tsuchiya H, Kanbe T, Harada K, Yashima K, et al. (2008) Aberrant expression of selenoproteins in the progression of colorectal cancer. Cancer Lett 259: 218-230.

14. Oberley TD, Oberley LW (1997) Antioxidant enzyme levels in cancer. Histo Histopathol 12: 525-535.

15. Saydam N, Kirb A, Demir O, Hazan E, Oto O, et al. (1997) Determination of glutathione, glutathione reductase, glutathione peroxidase and glutathione S-transferase levels in human lung cancer tissues. Cancer Lett 119: 13-19.

16. Tanriverdi T, Hanimoglu H, Kacira T, Sanus GZ, Kemerdere R, et al. (2007) Glutathione peroxidase, glutathione reductase and protein oxidation in patients with glioblastoma multiforme and transitional meningioma. J Cancer Res Clin Oncol 133: 627-633.

17. Kalinowski DS, Yu Y, Sharpe PC, Islam M, Liao YT, et al (2007) Design, synthesis, and characterization of novel iron chelators: structure-activity relationships of the 2-benzoylpyridine thiosemicarbazone series and their 3-nitrobenzoyl analogues as potent antitumor agents. J Med Chem 50: 37163729

18. Richardson DR, Sharpe PC, Lovejoy DB, Senaratne D, Kalinowski DS, et al. (2006) Dipyridyl thiosemicarbazone chelators with potent and selective antitumor activity form iron complexes with redox activity. J Med Chem 49 6510-6521.

19. Merlot AM, Kalinowski DS, Richardson DR (2012) Novel Chelators for Cancer Treatment: Where are we Now? Antioxid Redox Signal In Press: PMID:22424293.

20. Whitnall M, Howard J, Ponka P, Richardson DR (2006) A class of iron chelators with a wide spectrum of potent antitumor activity that overcomes resistance to chemotherapeutics. Proc Natl Acad Sci U S A 103: 14901-14906.

21. Yu Y, Rahmanto YS, Richardson DR (2011) Bp44mT: an orally active iron chelator of the thiosemicarbazone class with potent anti-tumour efficacy. $\mathrm{Br}$ Pharmacol 165: 148-166. 